\title{
Optimal Dynamic Pricing of Perishable Products with Reference Price Effect
}

\author{
Min WANG ${ }^{1, a,{ }^{*}, \text { Wen-Sheng YANG }}{ }^{1, b}$ \\ ${ }^{1}$ School of Economics and Management, Nanjing University of Science and Technology , Nanjing \\ 210094, China \\ a1483526528@qq.com, bwensheng_yang@163.com \\ *Min WANG
}

Keywords: Dynamic pricing, Strategic customer behavior, Reference price effect.

\begin{abstract}
This paper studies a two-stage dynamic pricing model with customers' strategic behavior and reference price effect. We used Stachelberg game theory and analyzed the decision-making of a monopolistic firm and its customers. Finally, we get the optimal pricing strategy for the firm in the market. What's more, the numerical analysis shows that the total profit is decreasing with the increase of the strategic customers' proportion. It also indicates that the optimal prices are increasing with the increase of the customers' memory coefficient. Results in this paper will give us some management implications.
\end{abstract}

\section{Introduction}

With the development of economic and internet, dynamic pricing is becoming more and more important in the marketing especially for perishable products. The value of this kind of products will decrease over time. Facing the dynamic pricing strategy of firms, more and more customers learn to choose right buying time to maximize their surplus. Su defined this kind of consumers as strategic customer; on the contrary, people who buy products without waiting for the sale price are defined as myopic customer [1]. Furthermore, Helson points out that customers' purchase decisions bases on the historical price posted by firms [2]. So we learn that not only do the costumers consider the current price, but also they compare it with the past price when they buy goods. Accordingly, the mental accounting framework provided by Thaler divides the total utility to two components: acquisition utility and transaction utility [3], which will be used in our paper. The latter part is the value of reference utility, so we can see that it is very important for us to consider the reference price effect in the dynamic pricing model. Below, we provide a brief literature review of dynamic pricing with strategic customers and reference dependence.

The consideration of strategic customers to the dynamic pricing problem is addressed by Besanko and Winston firstly in 1990 [4]. In recent years, researchers analyses various aspects of this problem. Xiao-feng LIU and Pei HUANG use the Stackelberg model and mechanism design to find optimal inventory and price under the strategic customers [5]. Mirko Kremer and Benny Mantin investigate the effect of customers' strategic behavior on the dynamic pricing in a set of behavioral experiments [6]. However, all this literature do not refer to the reference price effect, which is different to our paper. Other literature related to our paper study the problem on reference dependence. Huh proposes a two-stage multi-period negotiation model considering the customers who use historical price data to make their purchasing decision [7]. Hui YANG and Quan PAN build a two-period pricing model with customers' reference price. They finally get the optimal price strategies for the seller [8]. There are also some other papers discussing the reference prices, but they all ignore the existence of customers' strategic behavior.

Based on the researches above, our paper analyzes the dynamic pricing problem in the setting of customers' strategic behavior with reference price effect. We consider the reference price effect in the customers' utility function, which is different from literature we have listed firstly. Next, we formulate a demand function according to customers' strategic behavior using the distribution of customers' valuation, which is different from the general demand function $D(p, r)=a-b^{*} p+c^{*} x / r$ 
(where $c^{*} \mathrm{x} / \mathrm{r}$ refers to the reference effect). Last, we solve the model by inversion method of dynamic programming problem and obtain some useful conclusions.

\section{Model Description}

A profit-maximizing monopolistic firm sells a single product in a finite time horizon, $\mathrm{T}$. Demand in the market is certain, which means there are a pool of $\mathrm{N}$ risk-neutral customers. The proportion of strategic customers is $\alpha(0<\alpha<1)$ while that of myopic customers is $1-\alpha$. Each customer buys one unit of the product. We denote customers' values by uniform distribution in the interval [0, V] where $\mathrm{V}$ is the upper bound on the valuations. Each customer has known his own value while the firm and other customers only known the distribution of the value. The sequence of all events in Sales process is depicted in Fig. 1.

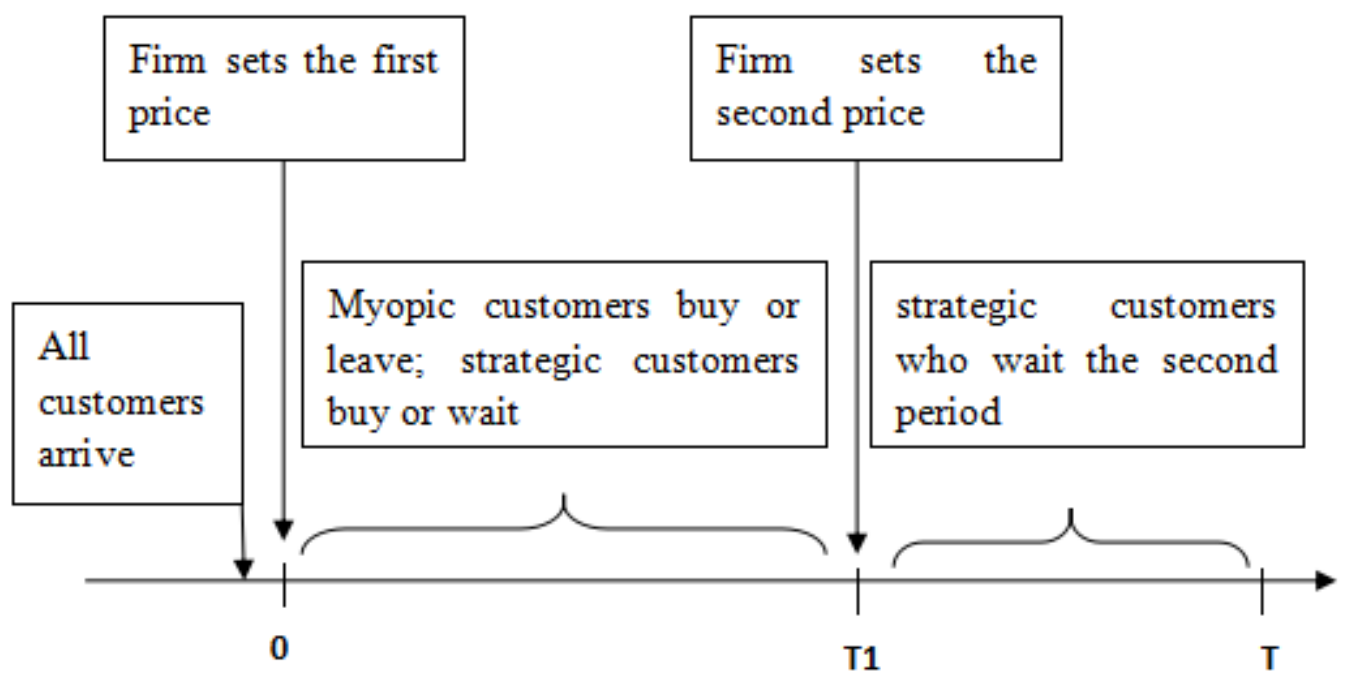

Fig. 1 Sequence of events in Sales process

The sales season is split into two sections, $\left[0, T_{1}\right]$ and $\left[T_{1}, T\right]$. All customers arrive at the beginning of the selling period. The monopolistic firm sets the first price $p_{1}$ at the start of the first stage. Myopic customers either buy the product or leave the market, which means they do not take part in the second stage [9]. On the contrary, strategic customers will have an expectation of the second price and decide when to buy in order to maximize their utility. The same to first stage, monopolistic firm chooses the second price $p_{2}$ (where $p_{1}>p_{2}$ ) at the beginning. Any strategic customers who wait for the sale price in this stage buy the product as long as their surplus is non-negative. There is a discounting factor $b$ $(0<b<1)$ on the customers' valuation because of perishable product itself or the waiting time. Furthermore, because the initial inventory is limited, customers waiting for the sale price will face the shortage risk, so we denote expected probability that customers can buy the product in the second stage by c $(0<\mathrm{c}<1)$.

Customers make the purchasing decision by weighting their utility in consideration of the reference price effect. To describe this reference effect, we refer to the expectation framework proposed by Nerlvove [10]. The specific form is as followed: $r_{t}=\gamma r_{t-1}+(1-\gamma) p_{t-1}$, Where $\gamma \in[0,1]$ represents the memory coefficient. We can see that the reference price is a weighted average of the reference price and posted price of the previous period.

Two basic assumptions in our paper are as followed: (1) the unit cost of the product is assumed to be zero for the convenience of calculation. (2) In the stage of $\left[0, T_{1}\right]$, Products are sold at first and customers have no history reference price, so that we do not consider the reference price effect in the 
first stage. In the second stage of $\left[T_{1}, T\right]$, customers often make decisions according to the price setting in the first stage, so that we have $r_{2}=p_{1}$.

\section{The Model and Optimal Policy}

We describe the customers' utility referring to the theory of mental accounting framework provided by Thaler [3]. So it is define as followed: $u(p)=(v-p)+\gamma(r-p)$. The first part $v-p$ is customers' acquisition utility, and the second part $\gamma(r-p)$ means the reference utility.

(1)The first stage $\left[0, T_{1}\right]$

Myopic customers' utility in this stage is $\left(v-p_{1}\right)$. They will buy the product when their surplus is above zero. Let $v_{1 m}$ denote the critical value of myopic customer in the first stage. We can get that $v_{1 m}=p_{1}$ and the demand function is $D_{1 m}=(1-\alpha) N \frac{V-v_{1 m}}{V}$.

Different from the myopic customers, strategic customers choose the maximum utility of the two stages. We make $v-p_{1}=c\left[\left(b v-p_{2}\right)+\gamma\left(r_{2}-p_{2}\right)\right]$ to find the critical value. Let $v_{1 s}$ denote the critical value of strategic customer in the first stage. We calculate that $v_{1 s}=\frac{p_{1}-c p_{2}+c \gamma\left(r_{2}-p_{2}\right)}{1-c b}$, and strategic customers prefer to buy the product in the first stage rather than wait when $v \geq v_{1 s}$. The demand function is: $D_{1 s}=\alpha N \frac{V-v_{1 s}}{V}$. So we have known that the total demand in the first stage is:

$$
\text { Eq. } 1 D_{1}=D_{1 m}+D_{1 s}=(1-\alpha) N \frac{V-v_{1 m}}{V}+\alpha N \frac{V-v_{1 s}}{V}
$$

(2)The second stage $\left[T_{1}, T\right]$

Any strategic customers waiting for the sale price will buy in this stage when $\left(v-p_{2}\right)+\gamma\left(p_{1}-p_{2}\right) \geq 0$ and $v \leq v_{1 s}$. As myopic customers do not participate in the second stage, the total demand in this stage is:

$$
\text { Eq.2 } D_{2}=D_{2 s}=\alpha N \frac{v_{1 s}-p_{2}+\gamma\left(p_{1}-p_{2}\right)}{V}
$$

Monopolistic firm is always profit-maximizing and strategic firm will make long-term decision. In the following section, we solve the problem by inversion method in dynamic programming problem.

(1) The second stage $\left[T_{1}, T\right]$

The profit of the second stage is: $R_{2}=p_{2} D_{2}=p_{2} \alpha N \frac{v_{1 s}-p_{2}+\gamma\left(p_{1}-p_{2}\right)}{V}$

Let $\frac{\partial R_{2}}{\partial p_{2}}=0$, and get the optimal condition:

$$
\text { Eq.3 } p_{2}^{*}=\frac{1}{2} \frac{-1-c \gamma-\gamma+\gamma c b}{-c-c \gamma-1+c b-\gamma+\gamma c b} p_{1}
$$

We can see that $p_{2}$ will be set by giving the price $p_{1}$ and related parameters.

(2) The first stage $\left[0, T_{1}\right]$

The profit of the first stage is: $R_{1}=p_{1} D_{1}=p_{1}\left[(1-\alpha) N \frac{V-v_{1 m}}{V}+\alpha N \frac{V-v_{1 s}}{V}\right]$.

So the total profit of the two stages is: 


$$
\text { Eq.4 } R=R_{1}+R_{2}=p_{1}\left[(1-\alpha) N \frac{V-v_{1 m}}{V}+\alpha N \frac{V-v_{1 s}}{V}\right]+p_{2} \alpha N \frac{v_{1 s}-p_{2}+\gamma\left(p_{1}-p_{2}\right)}{V}
$$

Where: we use $p_{2}^{*}$ in the formula (3) to replace $p_{2}$.

Let $\frac{\partial R}{\partial p_{1}}=0$, and get the optimal condition:

$$
\text { Eq.5 } p_{1}^{*}=\frac{-2 V(-c-c \gamma-1+c b-\gamma+\gamma c b)(-1+c b)}{-4+m_{1} c b-4 c \gamma+m_{2} c^{2} b-6 a c(\gamma+1)-\left(2 \gamma+\gamma^{2}\right) a c^{2}-6 a \gamma+a \gamma^{2}+m_{3} c^{2} b^{2}-4 c-4 \gamma-7 a}
$$

Where $m_{1}=12 a+8 \gamma+8+10 a \gamma-2 a \gamma^{2}, m_{2}=4 \gamma+6 a \gamma+4 a+4, m_{3}=a \gamma^{2}-4-4 a-4 \gamma-4 a \gamma$.

The firm can set the price $p_{1}$ by giving the related parameters above. Then use $p_{1}^{*}$ to replace $p_{1}$ in the formula (3). Finally we can get the optimal price $\left(p_{1}^{*}, p_{2}{ }^{*}\right)$ and relevant profit is the long-term maximum profit.

\section{Numerical Analysis}

In order to apply our results to the specific cases, we assume $V=20, \gamma=0.3, \alpha=0.5, \mathrm{~b}=0.6, \mathrm{c}=0.5$, $\mathrm{N}=2000$. We get the optimal prices $\left(p_{1}{ }^{*}, p_{2}{ }^{*}\right)=(5.2,2.3)$, which a monopolistic firm can set. And the total profit is 5242.9 in this situation. The optimal pricing strategy is given above, and then we present the influence of different parameters on the optimal prices and profit.

(1)Assuming other parameters remain unchanged, we analyze the proportion of strategic customers $\alpha$ on the total profit R. Then, we can get Fig. 2.

Fig. 2 shows that the total profit is strictly decreasing with the increase of strategic customers' proportion $\alpha$. Strategic behaviors of customers make them to choose the best purchase time and maximum their utility. So with the appearance of this type of people that some of them may buy the product in the sale price, the total profit decreases certainly. So the monopolistic firm may take some actions to reduce customers' strategic behavior in order to improve the total profit.

(2)Assuming other parameters remain unchanged, we analyze the memory coefficient $\gamma$ on the optimal prices.Then, we can get Fig. 3 .

It is obviously that $p_{1}{ }^{*}>p_{2}{ }^{*}$ in the figure. Furthermore, Fig. 3 shows that the optimal prices increase with the increasing of the memory parameters. But the magnitude of this increase is not very great. Customers who buy in the second stage will have a feel of getting more benefits. Intuitively, the bigger reference dependence consumers have, the greater demand of the second stage obtains and the higher of optimal price $p_{2}{ }^{*}$. This is different from studies which ignore customers' strategic behavior.

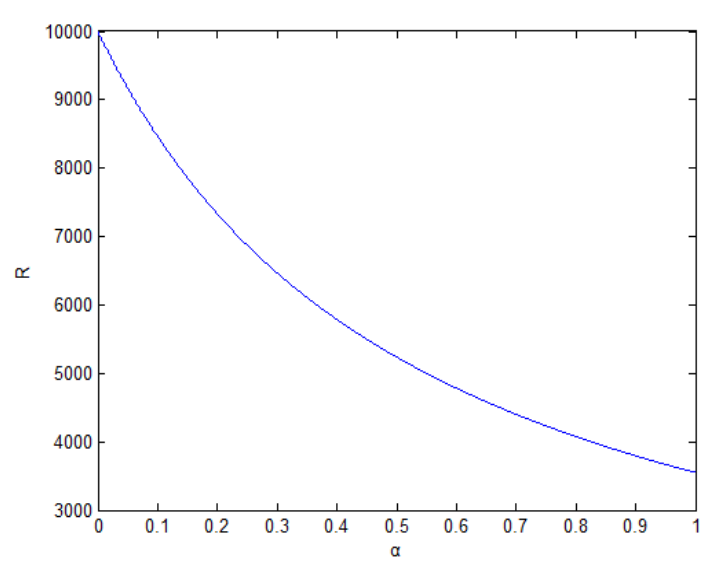

Fig.2 Effect of $\alpha$ on $\mathrm{R}$

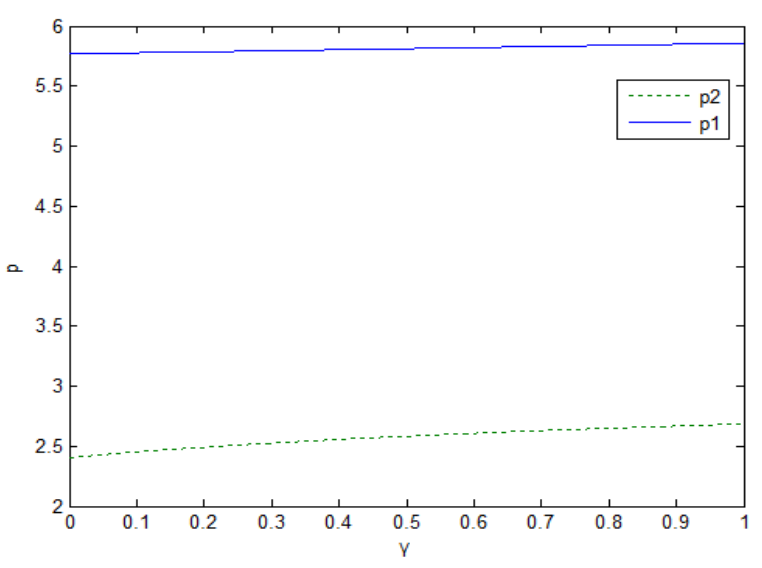

Fig. 3 Effect of $\gamma$ on $p_{1}{ }^{*}, p_{2}{ }^{*}$ 
(3)Assuming other parameters remain unchanged, we analyze the expected probability purchasing in the second stage on the total profit with two different situations $\alpha=0.1, \alpha=0.9$.Then, we can get Fig. 4.

We can see from Fig. 4 that the total profit decline with the increase of the expected probability. However, the magnitude of the manufacturer's profit changes more obviously when strategic consumers account for a larger proportion. Strategic customers are more likely to buy in the second stage when they think the opportunity buying in the second stage is large in order to maximum their utility. On the contrary, when myopic consumers in the market account for most, the total profit is larger and the effect of expected probability parameters on total profit is a little obviously.

(4)Assuming other parameters remain unchanged, we analyze the memory coefficient on the total profit with three different situations $\mathrm{c}=0.1, \mathrm{c}=0.6, \mathrm{c}=0.9$.Then, we can get Fig. 5 .

Fig. 5 indicates that effect of memory coefficient on the total profit depend on the value of the expected probability. When the probability that customers think they can buy the product in the second stage is high, they choose to wait for sale price and compare the price with the first stage. So the total profit is lower than the other two curves, and it is decreasing with the increase of the memory coefficient. While the probability purchasing in the sale price is just a little, any of them waiting for the sale price will buy the product quickly in the second stage. They regard that it is a rare opportunity to buy this product in this sale price comparing the full price. So the total profit is positively correlated to the parameter of memory as it is showed in the Fig. 5.

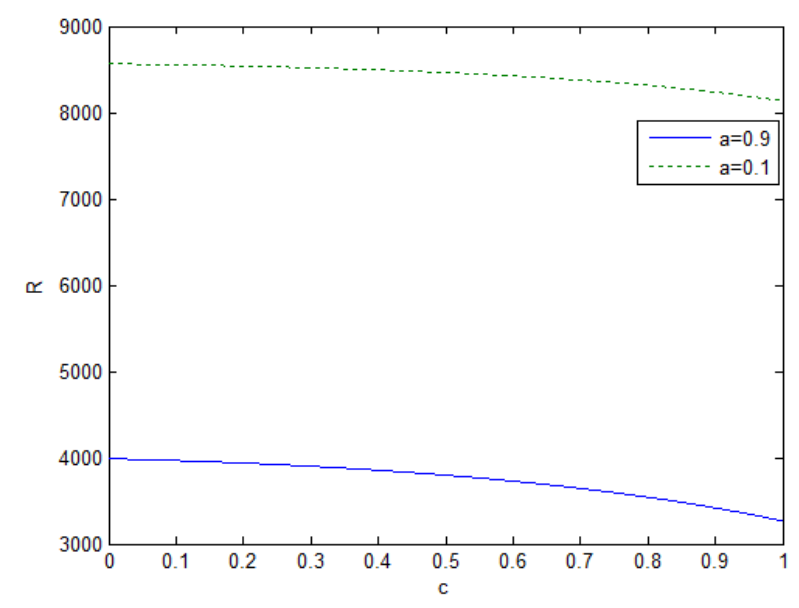

Fig. 4 Effect of c on $\mathrm{R}$

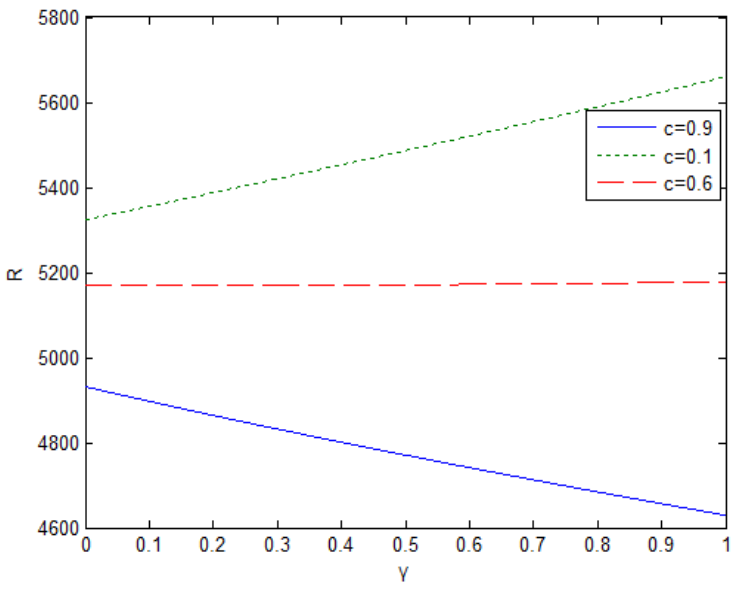

Fig. 5 Effect of $\gamma$ on $\mathrm{R}$

\section{Conclusion}

In this article, we formulate a two-stage dynamic pricing model under customers' strategic behavior considering reference price effect. Customers in our paper are divided into two kinds. One of them is myopic customers who buy the product when their surplus is above zero. Another kind is strategic customers who choose not only whether to buy but also when to buy the product. The most important is that we consider the influence of reference price when constructing the consumers' utility function. Finally, we solve the model by inversion method in dynamic programming problem and get the optimal prices for a profit-maximizing firm. The numerical analysis shows that the optimal prices are increasing with the increase of memory coefficient. But the Curve trend of total profit is distinct depending on different value of expected probability. So, to improve the total profit, firm can set up fewer initial inventories and make it public in order to reduce the expected probability that customers can buy in the second stage. Furthermore, firm can also take other actions to reduce the proportion of strategic customers to enhance their revenue as we can see in the figure. 
For future work, on one hand we can extend the model into a two-stage multi-period one. On the other hand we can build it in a competitive setting, which means two kinds products will be considered in the model. This will be interesting and get some more useful insights.

\section{References}

[1] Su X, Intertemporal pricing with strategic customer behavior, Management Science, 53(2007) 726-741.

[2] Helson, Harry, Adaptation-Level Theory, New York: Harper \& Row, (1964).

[3] R. Thaler, Toward a Positive Theory of Consumer Choice, Journal of Economic Behavior \& Organization, 1(1980) 39 60.

[4] Besanko, D., W.L.Winston, Optimal price skimming by a monopolist facing rational consumers, Management Science, 36(1990) 555 567.

[5] Xiao-feng LIU, Pei HUANG, Optimal dynamic pricing and inventory policy under strategic customers, Journal of Industrial Engineering and Engineering Management, 12(2009) 18 26, (In Chinese).

[6] Mirko Kremer, Benny Mantin, Dynamic Pricing in the Presence of Strategic Consumers: Theory and Experiment,. Social Science Electronic Publishing, (2015) 1-39.

[7] ]W. T. Huh, S. Kachani, et a1. A two-stage multi-period negotiation model with reference price effect, Journal of Revenue and Pricing Management, 9(2010) 443-475.

[8] Hui YANG, Quan PAN, A Two-period Pricing Model with Customers' Reference Prices, 2012 IEEE International Conference on Computer Science and Automation Engineering, (2012) 620-623, (In Chinese).

[9] Cachon G P, Swinney R, Purchasing, pricing, and quick response in the presence of strategic consumers, Management Science, 55(2009) 497-511.

[10] Nerlove, M, Adaptive expectations and cobweb phenomena, The Quarterly Journal of Economics, 72(1985) 227 240. 